using grindr@ at least once a month. MSM were significantly more likely to report having gonorrhoea and chlamydia (but not syphilis) in the past 12 months using grindr $($ at least once per month compared to never using grindr(t-test $=2.79$; $\mathrm{p}=0.003), \quad(\mathrm{t}$-test $=2.20 ; \mathrm{p}=0.028), \quad(\mathrm{t}$-tst $=0.58$; $\mathrm{p}=0.565)$.

Discussion/conclusion Use of the mobile phone application grindr $($ ) is associated with acquisition of bacterial STIs. Public health interventions to reduce STI rates in MSM should include using appropriate social media.

\section{UG3 ARE GEOSOCIAL NETWORKING (GSN) APPS ASSOCIATED WITH INCREASED RISK OF STIS \& HIV: A SYSTEMATIC REVIEW}

${ }^{1}$ Matheus Almeida*, 1,2Jo Gibbs, 'Claudia Estcourt. 'Queen Mary University of London, London, UK; 'niversity College London, London, UK

\subsection{6/sextrans-2016-052718.52}

Background/introduction Geosocial networking (GSN) apps such as Tinder and Grindr provide new ways of finding sex partners. It is suggested that usage could be responsible for increased STI \& HIV transmission.

Aim(s)/objectives To systematically review published literature to determine whether geosocial app use is associated with increased sexual risk behaviours, current and/or previous STIs \& HIV.

Methods Search of PubMed, EMBASE and Google Scholar for studies involving women, men, men who have sex with men (MSM) and use of GSN apps for sex-seeking which reported risk factors for STIs \& HIV transmission, published from 2009 to March 2016, in English. Search terms were associated using at least one regarding GSN apps and a second regarding STIs or sexual risk behaviours. Quality was assessed using Critical Appraisal Skills Programme criteria.

Results 13 studies met inclusion criteria: 12 cross-sectional studies, 1 review. All were in MSM from urban USA, China, Taiwan, UK and Ireland. In total there were 11924 subjects (range 927184). 7 studies reported app use to be associated with increased unprotected anal intercourse (UAI); 2 studies showed no association. 3 studies showed association with previous STI diagnoses, although association with HIV diagnoses had mixed results. 4 studies reported high response rate for app-based recruitment.

Discussion/conclusion Use of GSN apps is associated with factors known to facilitate STI \& HIV transmission in MSM. Studies in heterosexuals are much needed. High uptake of some apprecruited studies suggests GSN apps could be useful platforms for sexual health promotion and targeted risk reduction strategies.

\section{UG4 NON-SPECIFIC URETHRITIS: CAN WE BE A LITTLE MORE SPECIFIC?}

${ }^{1}$ Genevieve Hirst*, ${ }^{2}$ Daniel Richardson, ${ }^{2}$ Suneeta Soni. ${ }^{1}$ Peninsula Medical School, Truro, UK; ${ }^{2}$ Brighton and Sussex University Hospitals NHS Trust, Brighton, UK

\subsection{6/sextrans-2016-052718.53}

Background/introduction The causes of non-specific urethritis (NSU) in men are many and in GUM clinics, evidence for Chlamydia trachomatis (CT) and Neisseria gonorrhoeae (GC) is routinely sought. Mycoplasma genitalium (MG) accounts for 5-33\% of urethritis but is not routinely tested for in the UK. There is growing concern that widespread use of $1 \mathrm{~g}$ Azithromycin is leading to macrolide resistance in many organisms including MG.

Aim(s)/objectives To describe the current management of men with confirmed urethritis and their outcomes.

Methods Men with diagnoses of NSU from January to July 2015 were identified. Data were collected from electronic patient records. $\mathrm{p}$ values were obtained using chi-square test.

Results 254 cases of NSU were identified, median age 30 (range 16-69 years). 181/254 (71\%) heterosexual, 73/254 (29\%) MSM, 21/254 (8\%) HIV-positive. Rates of urethral CT and GC were $15 \%(n=40)$ and $1 \%(n=2)$ respectively. $21 / 254(8 \%)$ had persistent dysuria or discharge; $15 / 21$ of those were tested for MG; MG detected in 5/15 (33\%). Pathogens were identified in $17 \%$ of cases and heterosexual men were more likely to have pathogen-positive urethritis than MSM $(p=0.02)$. First line treatment: 93\% $1 \mathrm{~g}$ Azithromycin, 2.8\% doxycycline $100 \mathrm{mg}$ bd $7 / 7$.

Discussion/conclusion For the majority of NSU cases, no bacterial cause was identified yet these men were all prescribed antibiotics. MG was detected in a third of persistent NSU cases but may account for more as $1 \mathrm{~g}$ Azithromycin is enough to partially resolve symptoms but likely cause antimicrobial resistance. More effort should be made to determine the cause of urethritis in men so that appropriate antibiotics can be given where necessary.

\section{UG5 SH:24 - USER PERSPECTIVES ON AN ONLINE SEXUAL HEALTH SERVICE}

${ }^{1}$ Harriet Pittaway*, 'Sharmani Barnard, ${ }^{2}$ Emma Wilson, ${ }^{1}$ Paula Baraitser. 'King's Centre for Global Health, King's College London, London, UK; ${ }^{2}$ Department of Population Health, Faculty of Epidemiology and Population Health, London School of Hygiene and Tropical Medicine, London, UK

\subsection{6/sextrans-2016-052718.54}

Background/introduction The London Boroughs of Lambeth and Southwark have high levels of sexual health need and services are overstretched. SH:24 offers online testing for chlamydia, gonorrhoea, HIV and syphillis in Lambeth and Southwark, and the 'GetTested' randomised controlled trial evaluates its effectiveness.

Aim(s)/objectives This study aimed to document user views on clinic-based and online services.

Methods We analysed qualitative data from a follow-up questionnaire of the GetTested trial of 1337 participants, which included the following question: 'In your opinion, how could we improve the experience of getting a test from a sexual health service?' This data was quantitatively analysed against baseline characteristics to generate descriptive statistics. A thematic analysis of the free text responses was performed.

Results Three key themes were identified: interaction with services; ease of use and experienced stigma. A subjective variable was developed to describe whether users needs were met. More participants reported the online service as meeting their needs than the clinic service. Areas needing improvement identified within the clinic arm were: Information prior to service use, Improved confidentiality \& Waiting times. Areas needing improvement identified within the online arm were: Lack of personal contact, Difficulty with the self-sampling process, Confidence in ability to self-test.

Discussion/conclusion The problems identified with face-to-face services are overcome by online services and vice versa. In order 
to be successful, both need to work in collaboration to provide accessible and acceptable services.

\section{UG6 PLEASE DON'T TELL MY GP: PATIENTS' CONCERNS ABOUT THE SHARING OF INFORMATION BETWEEN SEXUAL HEALTH CLINICS AND GENERAL PRACTITIONERS (GPS)}

${ }^{1}$ Qiang Lu*, ${ }^{2}$ Emily Clarke, ${ }^{1,3}$ Raj Patel, ${ }^{1}$ Harriet Eatwell, ${ }^{1}$ Rohilla Maarij. ${ }^{1}$ School of Medicine, University of Southampton, Southampton, UK; ${ }^{2}$ Department of Sexual Health, Solent NHS Trust, St Mary's Community Health Campus, Portsmouth, UK; ${ }^{3}$ Department of Sexual Health, Solent NHS Trust, Royal South Hants Hospital, Southampton, UK

\subsection{6/sextrans-2016-052718.55}

Background/introduction At sexual health clinics, patients are asked for permission to contact them by a variety of methods. When patients who have opted-out of GP contact are found to have a sexually transmitted infection (STI) and cannot be contacted despite multiple attempts, a case-by-case decision is often made, regarding breaching the patient's permissions and contacting their GP.

Aim(s)/objectives To determine why some patients decline GP contact, and to assess their views on GP contact against their expressed wishes, in order to treat an STI, when a patient is unable to be contacted by other means.

Methods This was a prospective, qualitative, NRES-approved study involving 10 semi-structured interviews with patients attending a level 3 UK sexual health clinic who had declined GP contact.

Results Three key areas of concern were identified: potential negative implications of permanently recording sexual health problems on GP records, including the effect on future life insurance and job applications; concerns about receptionists in GP surgeries breaking confidentiality in the reception area and being judgmental; and patients' close relationship with their GP. However, 8/10 of those interviewed supported a breach of permissions by contacting their GP in order to treat an STI.

Conclusion With the increased involvement of GPs in delivering sexual health services in the UK, it is essential that action is taken to improve patients' confidence in confidentiality protections at their GP. Sexual health clinics should ensure they explain why GP contact may be required in order to potentially increase patients' willingness for this to occur.

\section{Section 5 Poster presentations}

\section{P001 IN 2015, MSM ACCESSING PEPSE IS SIGNIFICANTLY MORE ASSOCIATED WITH CLUB DRUG USE THAN 2013/2014}

${ }^{1}$ Zoe Ottaway*, ${ }^{2}$ Daniel Richardson. ${ }^{1}$ Maidstone and Tunbridge Wells NHS Trust, Maidstone, Kent, UK; ${ }^{2}$ Brighton and Sussex University Hospitals NHS Trust, Brighton, Sussex, UK

\subsection{6/sextrans-2016-052718.56}

Background/introduction Recreational drug (RD) use is increasing in men who have sex with men (MSM) and increases sexual risk taking behaviour and possibly increasing attendances for PEPSE.

Aim(s)/objectives To identify Club drug use during PEPSE attendances in MSM in 2013/4 compared to 2015.

Methods Review PEPSE (MSM) attendance during two 4-month periods: November 2013 to February 2014 and March 2015 to June 2015.

Results 152 MSM attended for PEPSE: 51 in 2013/4 and 101 in 2015. The median age was 31 (18-79) years. Documentation of Club drug use during PEPSE episode increased significantly from $27 / 51(53 \%)$ in $2013 / 14$ to $100 / 101$ (99\%) during 2015 ( $p<0.001)$. Club drug use during PEPSE episode increased significantly from $9 / 51(18 \%)$ in $2013 / 4$ to $41 / 101$ (41\%) in 2015 (OR 3.19, $\mathrm{p}<0.005)$. There were no significant changes in the Club drugs being used: gamma-Butyrolactone(GBL), Mephedrone and Crystal Meth being the most frequent reported.

Discussion/conclusion Episodes of unsafe sex leading to access of PEPSE appear to be more associated with club drug use in 2015 than in 2013/4 and our documentation of this has improved. Identification of club drug use in MSM is an important harm intervention.

\section{P002 IS ENQUIRY REGARDING ALCOHOL CONSUMPTION AND ALCOHOL REDUCTION ADVICE ACCEPTABLE TO SEXUAL HEALTH SERVICE USERS? A CROSS-SECTIONAL STUDY OF CLINIC ATTENDEES}

\footnotetext{
1,2Martyn Wood. 'Mid-Cheshire Hospitals NHS foundation Trust, Cheshire, UK; ${ }^{2}$ Royal Liverpool University Hospital, Liverpool, UK
}

10.1136/sextrans-2016-052718.57

Background/introduction Problem alcohol consumption is a major health problem in the UK. Alcohol assessment and behavioural advice or "brief interventions" are effective in decreasing alcohol intake in primary and secondary care but not in sexual health clinics.

Aim(s)/objectives We assessed sexual health service user views towards alcohol screening using a prospective cross-sectional survey to identify any themes, which limit acceptability of these methods.

Methods Age, gender, alcohol consumption measured by AUDIT-C score, and opinion towards 10 statements on alcohol screening within a sexual health clinic were assessed.

Results 462 surveys were returned. Respondents were 64\% female, $36 \%$ male. Most, $53.7 \%$, were aged $\geq 25$ years, the highest number of responses was received from those aged 20-24 (32.2\%), median age category was 25-29 years. The majority of respondents, $61.6 \%$ had hazardous alcohol consumption. Males had more positive AUDIT-C scores (indicating hazardous alcohol consumption) compared to females (75\% vs 54\%, p < 0.001 ). Those aged $<30$ had more positive AUDIT-C scores $(67.9 \%$ vs $32.1 \%, \mathrm{p}<0.001)$. Attitudes to alcohol assessment performed by sexual health practitioners were positive (range 91.1\%$74.5 \%$ favourable), responses were less favourable, becoming negative towards the appropriateness of the sexual health clinic as a screening venue (range 56.7\%-33.6\% favourable). Responses to 4 out of 10 opinion statements were related (multivariate regression model) to age or AUDIT-C score.

Discussion/conclusion Different strategies need to be explored within sexual health for alcohol consumption reduction 